\title{
Dispersive approach to non-Abelian axial anomaly
}

\author{
Sergey Khlebtsov, ${ }^{1}$ Yaroslav Klopot,${ }^{2,3, *}$ Armen Oganesian, ${ }^{1,2, \uparrow}$ and Oleg Teryaev ${ }^{2, *}$ \\ ${ }^{1}$ Institute of Theoretical and Experimental Physics, 117218 Moscow, Russia \\ ${ }^{2}$ Joint Institute for Nuclear Research, 141980 Dubna, Russia \\ ${ }^{3}$ Bogolyubov Institute for Theoretical Physics, 03143 Kiev, Ukraine
}

(Received 14 June 2018; published 8 January 2019)

\begin{abstract}
Manifestations of strong and electromagnetic axial anomalies in two-photon decays of $\eta$ and $\eta^{\prime}$ mesons are studied. Applying a dispersive approach to axial anomaly in the singlet current, we obtain an anomaly sum rule containing strong and electromagnetic anomaly contributions. The relevant low-energy theorem was generalized to the case of mixed states and used to evaluate the subtraction constant of the strong anomaly-related form factor $\langle 0|G \tilde{G}| \gamma \gamma\rangle$. We made a numerical estimation of the contributions of gluon and electromagnetic anomalies to the two-photon decays of $\eta$ and $\eta^{\prime}$ mesons and found significant suppression of the gluon anomaly contribution.
\end{abstract}

DOI: 10.1103/PhysRevD.99.016008

\section{INTRODUCTION}

Axial (chiral) anomaly [1,2]—violation of the axial symmetry of classical theory by quantum fluctuationsis an important phenomenon inherent to QCD with many interesting consequences. In particular, axial anomaly is known to play an essential role in the two-photon decays of pseudoscalar mesons. As a matter of fact, it was the pion decay problem that led to the discovery of quantum anomalies. Precision measurements of two-photon decays of $\pi^{0}[3,4], \eta$, and $\eta^{\prime}$ mesons remain a unique tool for the study of properties of QCD and effective theories at the low-energy limit, including such subtle effects as chiral symmetry breaking and mixing.

Besides its connection with the real photon processes, the axial anomaly is intimately connected with the processes involving virtual photons: the dispersive form of the axial anomaly [5] (for a review, see e.g., [6]) leads to the anomaly sum rules (ASRs) [7-9] which can be used to evaluate the photon-meson transitions $\gamma(k) \gamma^{*}(q) \rightarrow$ $\pi^{0}\left(\eta, \eta^{\prime}\right)$ at arbitrary photon virtuality. This approach was used to study the transition form factors of the $\pi^{0}, \eta$ and $\eta^{\prime}$ mesons in the spacelike [10-15] and timelike [16] regions. Along with the study based on the ASRs, the transition form factors have been a subject of extensive investigation

\footnotetext{
*klopot@theor.jinr.ru

†armen@itep.ru

*teryaev@theor.jinr.ru
}

Published by the American Physical Society under the terms of the Creative Commons Attribution 4.0 International license. Further distribution of this work must maintain attribution to the author(s) and the published article's title, journal citation, and DOI. Funded by SCOAP ${ }^{3}$. within other frameworks recently, such as light cone sum rules [17-19], constituent [20], light-front [21] and nonlocal chiral quark models [22], light-front holographic QCD [23] as well as in some other models [24-26] and model-independent analyses [27-29].

The presence of the axial anomaly results in the nonconservation of the axial current (even in the chiral limit). For the axial current $J_{\mu 5}=\bar{\psi}_{i} \gamma_{\mu} \gamma_{5} \psi_{i}$, where $\psi_{i}$ is some quark field of unit charge $e$, the axial anomaly leads to

$$
\partial^{\mu} J_{\mu 5}=2 i m_{i} \bar{\psi}_{i} \gamma_{5} \psi_{i}+\frac{e^{2}}{8 \pi^{2}} N_{c} F \tilde{F}+\frac{\alpha_{s}}{4 \pi} G \tilde{G}
$$

where $F$ and $G$ are electromagnetic and gluon field strength tensors, respectively, $\tilde{F}^{\mu \nu}=\frac{1}{2} \epsilon^{\mu \nu \rho \sigma} F_{\rho \sigma}$ and $\tilde{G}^{\mu \nu, a}=\frac{1}{2} \epsilon^{\mu \nu \rho \sigma} G_{\rho \sigma}^{a}$ are their duals, $N_{c}=3$ is a number of colors, $\alpha_{s}$ is a strong coupling constant.

In the case of light pseudoscalar mesons the relevant currents are the diagonal components of the octet of axial currents $J_{\mu 5}^{(a)}=(1 / \sqrt{2}) \sum_{i} \bar{\psi}_{i} \gamma_{\mu} \gamma_{5} \lambda^{a} \psi_{i}$ and the singlet axial current $J_{\mu 5}^{(0)}=(1 / \sqrt{3}) \sum_{i} \bar{\psi}_{i} \gamma_{\mu} \gamma_{5} \psi_{i}$, where the sum is over the flavors of light quarks $i=u, d, s, \lambda^{a}$ are the diagonal Gell-Mann $S U(3)$ matrices, $a=3,8$. While the $\pi^{0}$ is almost a pure $S U(3)$ flavor state (with corresponding $J_{\mu 5}^{(3)}$ current), the $\eta$ and $\eta^{\prime}$ mesons are not-their physical states are a significant mixture of the octet and singlet $S U(3)$ states (related to $J_{\mu 5}^{(8)}$ and $J_{\mu 5}^{(0)}$ currents). The mixing in the $\eta-\eta^{\prime}$ system results in four nonzero decay constants $f_{M}^{(i)}$, defined as the currents' projections onto meson states $M\left(i=8,0 ; M=\eta, \eta^{\prime}\right)$, 


$$
\left\langle 0\left|J_{\mu 5}^{(i)}(0)\right| M(p)\right\rangle=i p_{\mu} f_{M}^{i} .
$$

It is important that the octet of axial currents is free from the strong (gluon) anomaly part while the singlet axial current acquires both electromagnetic as well as gluon anomalies,

$\partial^{\mu} J_{\mu 5}^{(a)}=\frac{2 i}{\sqrt{2}} \sum_{i} m_{i} \bar{\psi}_{i} \gamma_{5} \lambda^{a} \psi_{i}+\frac{e^{2}}{8 \pi^{2}} C^{(a)} N_{c} F \tilde{F}, \quad a=3,8$,

$\partial^{\mu} J_{\mu 5}^{(0)}=\frac{2 i}{\sqrt{3}} \sum_{i} m_{i} \bar{\psi}_{i} \gamma_{5} \psi_{i}+\frac{e^{2}}{8 \pi^{2}} C^{(0)} N_{c} F \tilde{F}+\frac{\sqrt{3} \alpha_{s}}{4 \pi} G \tilde{G}$,

where $C^{(a)}$ are the charge factors $\left(e_{i}\right.$ are quark charges in units of the electron charge):

$$
\begin{aligned}
& C^{(3)}=\frac{1}{\sqrt{2}}\left(e_{u}^{2}-e_{d}^{2}\right)=\frac{1}{3 \sqrt{2}}, \\
& C^{(8)}=\frac{1}{\sqrt{6}}\left(e_{u}^{2}+e_{d}^{2}-2 e_{s}^{2}\right)=\frac{1}{3 \sqrt{6}}, \\
& C^{(0)}=\frac{1}{\sqrt{3}}\left(e_{u}^{2}+e_{d}^{2}+e_{s}^{2}\right)=\frac{2}{3 \sqrt{3}} .
\end{aligned}
$$

Absence of the gluon anomaly for the third (isovector) and the eighth (octet) components of the octet of axial currents allowed to derive the anomaly sum rules $[10,11,13,14,16]$ which benefited from the absence of corrections due to Adler-Bardeen theorem and t'Hooft's principle.

The singlet axial current has a complication due to gluon anomaly part. This paper is aimed to investigate this issue. We derive the anomaly sum rule based on the dispersive form of axial anomaly in the singlet channel and study the contributions of electromagnetic and gluon parts of the axial anomaly to the two-photon decays of the $\eta$ and $\eta^{\prime}$ mesons.

The paper is organized as follows. In Sec. II, we derive the anomaly sum rule for the singlet axial current. In Sec. III, we generalize the low-energy theorem for the case of mixing $\left(\eta-\eta^{\prime}\right)$ states. In Sec. IV, we apply the results of the previous sections to study the role of electromagnetic and gluon parts of the axial anomaly in the meson decays.

\section{DISPERSIVE APPROACH TO AXIAL ANOMALY WITH A GLUON TERM}

In order to study the hadron observables in the nonperturbative region, we will develop a sum rule based on the dispersive representation of the axial anomaly in the singlet current. Consider the triangle graph amplitude, composed of the axial current $J_{\alpha 5}$ with momentum $p=$ $k+q$ and two vector currents with momenta $k$ and $q$,

$$
\begin{aligned}
& \int d^{4} x d^{4} y e^{(i k x+i q y)}\left\langle 0\left|T\left\{J_{\alpha 5}(0) J_{\mu}(x) J_{\nu}(y)\right\}\right| 0\right\rangle \\
& =e^{2} T_{\alpha \mu \nu}(k, q) .
\end{aligned}
$$

This amplitude can be decomposed [30] (see also [31,32]) as

$$
\begin{aligned}
T_{\alpha \mu \nu}(k, q)= & F_{1} \varepsilon_{\alpha \mu \nu \rho} k^{\rho}+F_{2} \varepsilon_{\alpha \mu \nu \rho} q^{\rho} \\
& +F_{3} k_{\nu} \varepsilon_{\alpha \mu \rho \sigma} k^{\rho} q^{\sigma}+F_{4} q_{\nu} \varepsilon_{\alpha \mu \rho \sigma} k^{\rho} q^{\sigma} \\
& +F_{5} k_{\mu} \varepsilon_{\alpha \nu \rho \sigma} k^{\rho} q^{\sigma}+F_{6} q_{\mu} \varepsilon_{\alpha \nu \rho \sigma} k^{\rho} q^{\sigma},
\end{aligned}
$$

where the coefficients $F_{j}=F_{j}\left(p^{2}, k^{2}, q^{2} ; m^{2}\right), j=1, \ldots, 6$ are the corresponding Lorentz invariant amplitudes constrained by current conservation and Bose symmetry. Note that the latter includes the interchange $\mu \leftrightarrow \nu, k \leftrightarrow q$ in the tensor structures and $k^{2} \leftrightarrow q^{2}$ in the arguments of the scalar functions $F_{j}$.

The anomalous axial-vector Ward identity for $T_{\alpha \mu \nu}(k, q)$ for the singlet axial current $J_{\mu 5}^{(0)}(p)$ and photons $\gamma\left(k, \epsilon^{(k)}\right)$, $\gamma\left(q, \epsilon^{(q)}\right)$ (real or virtual) reads

$$
\begin{aligned}
p_{\alpha} T^{\alpha \mu \nu}= & 2 \sum_{i} m_{i} G_{i} \epsilon^{\mu \nu \rho \sigma} k_{\rho} q_{\sigma}+\frac{C^{(0)} N_{c}}{2 \pi^{2}} \epsilon^{\mu \nu \rho \sigma} k_{\rho} q_{\sigma} \\
& +N\left(p^{2}, q^{2}, k^{2}\right) \epsilon^{\mu \nu \rho \sigma} k_{\rho} q_{\sigma},
\end{aligned}
$$

where the sum is over $i=u, d, s$ and

$\left\langle 0\left|\frac{1}{\sqrt{3}} \sum_{i} m_{i} \bar{\psi}_{i} \gamma_{5} \psi_{i}\right| \gamma \gamma\right\rangle=2 \sum_{i} m_{i} G_{i} \epsilon^{\mu \nu \rho \sigma} k_{\rho} q_{\sigma} \epsilon_{\rho}^{(k)} \epsilon_{\sigma}^{(q)}$,

$\left\langle 0\left|\frac{\sqrt{3} \alpha_{s}}{4 \pi} G \tilde{G}\right| \gamma \gamma\right\rangle=e^{2} N\left(p^{2}, k^{2}, q^{2}\right) \epsilon^{\mu \nu \rho \sigma} k_{\mu} q_{\nu} \epsilon_{\rho}^{(k)} \epsilon_{\sigma}^{(q)}$,

$\langle 0|F \tilde{F}| \gamma \gamma\rangle=2 \epsilon^{\mu \nu \rho \sigma} k_{\mu} q_{\nu} \epsilon_{\rho}^{(k)} \epsilon_{\sigma}^{(q)}$.

We introduced here the form factors $G_{i}$ and $N$, while the last matrix element is pointlike up to QED corrections.

In the kinematical configuration with one real photon $\left(k^{2}=0\right)$ which we consider in the rest of this section, the above anomalous Ward identity can be rewritten in terms of form factors $F_{j}, G_{i}, N$ as follows $\left(N\left(p^{2}, q^{2}\right) \equiv\right.$ $\left.N\left(p^{2}, q^{2}, k^{2}=0\right)\right)$ :

$$
\left(q^{2}-p^{2}\right) F_{3}-q^{2} F_{4}=\sum_{i} 2 m_{i} G_{i}+\frac{C^{(0)} N_{c}}{2 \pi^{2}}+N\left(p^{2}, q^{2}\right) .
$$


We can write the form factors $G_{i}, F_{3}, F_{4}$ as dispersive integrals without subtractions. Indeed, in the case of isovector and octet channels (free from gluon anomaly) it can be shown explicitly [8]. In the considered case of the singlet current from simple dimensional arguments one can assume that $G_{i}, F_{3,4}$ decrease at large $p^{2}$, and, therefore, the form factors can be written as dispersive integrals without subtractions. On the other hand, generally speaking, one should get the subtraction constant in the dispersion relation for the form factor $N$, analogous to the Abelian anomaly constant $\frac{C^{(0)} N_{c}}{2 \pi^{2}}$. Let us rewrite this dispersion relation in the form with one subtraction at $p^{2}=0$ :

$$
N\left(p^{2}, q^{2}\right)=N\left(0, q^{2}\right)+p^{2} R\left(p^{2}, q^{2}\right),
$$

where the new form factor $R$ can be written as an unsubtracted dispersive integral. Then the imaginary part of (14) with respect to $p^{2}$ ( $s$ in the complex plane) reads

$$
\left(q^{2}-s\right) \operatorname{Im} F_{3}-q^{2} \operatorname{Im} F_{4}=2 \sum_{i} m_{i} \operatorname{Im} G_{i}+s \operatorname{Im} R
$$

Dividing every term of Eq. (16) by $\left(s-p^{2}\right)$ and integrating over $s \in[0,+\infty)$, we get $^{1}$

$$
\begin{aligned}
& \frac{1}{\pi} \int_{0}^{\infty} \frac{\left(q^{2}-s\right) \operatorname{Im} F_{3}}{s-p^{2}} d s-\frac{q^{2}}{\pi} \int_{0}^{\infty} \frac{\operatorname{Im} F_{4}}{s-p^{2}} d s \\
& \quad=\frac{1}{\pi} \sum_{i} \int_{0}^{\infty} \frac{2 m_{i} \operatorname{Im} G_{i}}{s-p^{2}} d s+\frac{1}{\pi} \int_{0}^{\infty} \frac{s \operatorname{Im} R}{s-p^{2}} d s .
\end{aligned}
$$

After simple transformation of the first and last terms in (17) and making use of the dispersive relations for the form factors $F_{3}, F_{4}, G_{i}$, $R$, we arrive at

$$
\begin{aligned}
& \left(q^{2}-p^{2}\right) F_{3}-\frac{1}{\pi} \int_{0}^{\infty} \operatorname{Im} F_{3} d s-q^{2} F_{4} \\
& =2 \sum_{i} m_{i} G_{i}+p^{2} R+\frac{1}{\pi} \int_{0}^{\infty} \operatorname{Im} R d s .
\end{aligned}
$$

Comparing now (18) with (14), we can write down the anomaly sum rule for the singlet current:

$\frac{1}{\pi} \int_{0}^{\infty} \operatorname{Im} F_{3} d s=\frac{C^{(0)} N_{c}}{2 \pi^{2}}+N\left(0, q^{2}\right)-\frac{1}{\pi} \int_{0}^{\infty} \operatorname{Im} R\left(s, q^{2}\right) d s$,

Saturating the lhs of (19) with resonances according to global quark-hadron duality, we write out the first

\footnotetext{
${ }^{1}$ The lower limits of the integrals are formally expressed in terms of quark masses, but due to confinement they should be replaced with a pion mass (see, e.g., [33]), which we neglect anyway.
}

resonances' contributions explicitly, while the higher states are absorbed by the integral with a lower limit $s_{0}$,

$$
\begin{aligned}
& \sum_{M} f_{M}^{0} F_{M \gamma}\left(q^{2}\right)+\frac{1}{\pi} \int_{s_{0}}^{\infty} \operatorname{Im} F_{3} d s \\
& =\frac{C^{(0)} N_{c}}{2 \pi^{2}}+N\left(0, q^{2}\right)-\frac{1}{\pi} \int_{0}^{\infty} \operatorname{Im} R\left(s, q^{2}\right) d s,
\end{aligned}
$$

where the hadron contributions are expressed in terms of the decay constants $f_{M}^{0}(2)$ and form factors $F_{M \gamma}\left(q^{2}\right)$ of the transitions $\gamma \gamma^{*} \rightarrow M$

$$
\int d^{4} x e^{i k x}\left\langle M(p)\left|T\left\{J_{\mu}(x) J_{\nu}(0)\right\}\right| 0\right\rangle=e^{2} \epsilon_{\mu \nu \rho \sigma} k^{\rho} q^{\sigma} F_{M \gamma}\left(q^{2}\right) .
$$

Let us make a significant note about derivation of Eqs. (19) and (20). Due to multiplicative renormalization of matrix element of axial current and corresponding operators [1,34], in anomaly Ward identity the scale dependence is the same for all terms in left and right sides of Eq. (10).

Then, bearing in mind that renormalization should not change a power behavior at infinity of corresponding invariant amplitudes, we may conclude, that dispersive relations without subtractions for invariant amplitude still remain fair. As a result, derivation of Eq. (19) remains valid at any scale, so that it does not depend of normalization point.

The problem of normalization point appears, however, in Eq. (20) which was obtained from Eq. (19) by use of quarkhadron duality. In this case, clearly, when one saturates the imaginary part by resonance contributions, the decay constants are fixed at corresponding resonance masses. So, in Eq. (20), the normalization point for decay constants $f_{M}^{0}$ in first approximation can be taken at $\eta^{\prime}$ mass scale, which is a typical mass scale for singlet current.

The lower limit $s_{0}\left(q^{2}\right)$ in the integral in the lhs of (20) should range between the masses squared of the last taken into account resonance and the first resonance included into the integral term. The choice of $s_{0}$ for the isovector and octet channels was discussed earlier [12,15]. For the case of singlet current, keeping $\eta$ and $\eta^{\prime}$ mesons in the first term of (20), we expect $s_{0} \gtrsim 1 \mathrm{GeV}^{2}$. Actually, this estimation is sufficient for the purposes of the present paper.

As a note, let us point out the following observation. We can also saturate with resonances the last term in the ASR (19). The main contributions are given, in particular, by the glueball-like states. Although it is hard to draw any numerical conclusions at present (for instance, the decay constants of the respective states are not known), the ASR can be useful for estimation of their relative contributions in the future. 


\section{LOW-ENERGY THEOREM AND MIXING}

An important part of the ASR (20), representing gluon anomaly, is related to the matrix element $\langle 0|G \tilde{G}(p)| \gamma(k) \gamma(q)\rangle$. Rigorous QCD calculation of this matrix element encounters difficulties due to confinement and is not known yet. However, it is possible to estimate it in the limit $p^{\mu}=0$. Hereafter, we consider the case of two real photons $\left(q^{2}=k^{2}=0\right)$.

The idea is simple (see [35] and references therein). Consider the matrix element of the singlet axial current $\left\langle 0\left|J_{\mu 5}^{(0)}(p)\right| \gamma \gamma\right\rangle$. Supposing that there are no massless particles in the singlet channel in the chiral limit, as the $\eta^{\prime}$ meson remains massive, one must get $\lim _{p \rightarrow 0} p^{\mu}\left\langle 0\left|J_{\mu 5}(p)\right| \gamma \gamma\right\rangle=0$. This corresponds to $\left\langle 0\left|\partial^{\mu} J_{\mu 5}\right| \gamma \gamma\right\rangle=0$, so using the explicit expression for the divergence of axial current in the chiral limit (put $m_{q}=0$ ), one can relate the matrix elements of $\langle 0|G \tilde{G}| \gamma \gamma\rangle$ and $\langle 0|F \tilde{F}| \gamma \gamma\rangle$ in the considered limits.

However, due to a significant mixing in the $\eta-\eta^{\prime}$ system, the assumption of [35], that the singlet channel in the chiral limit does not contain massless particles, is violated by the contribution of the massless in the chiral limit $\eta$. Therefore, our aim now is to construct such a current, that has no projections onto the Goldstone states. Taking into account that $\pi^{0}$ meson has a negligible projection onto $J_{\mu 5}^{(8)}$ and $J_{\mu 5}^{(0)}(\sim 1 \%[36,37])$, we can limit our basis to these currents and require the current to be orthogonal only to $\eta$ :

$$
J_{\mu 5}^{(X)}=a J_{\mu 5}^{(0)}+b J_{\mu 5}^{(8)}, \quad\left\langle 0\left|J_{\mu 5}^{(X)}\right| \eta\right\rangle=0 .
$$

After eliminating the constant $a$, in terms of meson decay constants this current reads

$$
J_{\mu 5}^{(X)}=b\left(J_{\mu 5}^{(8)}-\frac{f_{\eta}^{8}}{f_{\eta}^{0}} J_{\mu 5}^{(0)}\right)
$$

where $\mathrm{b}$ is an arbitrary constant and the decay constants $f_{M}^{(i)}$ are the defined in (2). The current (23) gives no massless poles in the matrix element $\left\langle 0\left|J_{\mu 5}^{(x)}\right| \gamma \gamma\right\rangle$ even in the chiral limit, so

$$
\lim _{p \rightarrow 0}\left\langle 0\left|\partial_{\mu} J_{\mu 5}^{(X)}(p)\right| \gamma \gamma\right\rangle=0 .
$$

Using explicit expressions for the divergences of currents in the chiral limit, at $p^{\mu}=0$ we immediately obtain the following relation between the matrix elements of $G \tilde{G}$ and $F \tilde{F}$ :

$\left\langle 0\left|\frac{\sqrt{3} \alpha_{s}}{4 \pi} G \tilde{G}\right| \gamma \gamma\right\rangle=\frac{N_{c}}{f_{\eta}^{8}}\left(f_{\eta}^{0} C^{(8)}-f_{\eta}^{8} C^{(0)}\right)\left\langle 0\left|\frac{\alpha_{e}}{2 \pi} F \tilde{F}\right| \gamma \gamma\right\rangle$.
This gives us the value of the subtraction constant of the gluon anomaly,

$$
N(0,0,0)=\frac{N_{c}}{2 \pi^{2} f_{\eta}^{8}}\left(f_{\eta}^{0} C^{(8)}-f_{\eta}^{8} C^{(0)}\right) .
$$

\section{HADRON CONTRIBUTIONS AND ANALYSIS OF THE ASR}

As we mentioned above, the first hadron contributions to the ASR (20) are given by $\eta$ and $\eta^{\prime}$. We keep these resonances as explicit contributions, while the rest of the resonances are absorbed by the integral "continuum" term. In what follows, we limit ourselves to the case of real photons, i.e., $k^{2}=q^{2}=0$. In this limit the transition form factors determine the two-photon decay amplitudes $A_{M}$ $\left(M=\eta, \eta^{\prime}\right)$ which are expressed in terms of the decay widths of the mesons $\Gamma_{M \rightarrow 2 \gamma}$ as follows:

$$
A_{M} \equiv F_{M \gamma}(0)=\sqrt{\frac{64 \pi \Gamma_{M \rightarrow 2 \gamma}}{e^{4} m_{M}^{3}}} .
$$

Recall also, that the ASR for the octet channel [14] in the case of real photons leads to

$$
f_{\eta}^{8} A_{\eta}+f_{\eta^{\prime}}^{8} A_{\eta^{\prime}}=\frac{1}{2 \pi^{2}} N_{c} C^{(8)} .
$$

The ASR (20) for the singlet channel for real photons can be written as follows:

$$
f_{\eta}^{0} A_{\eta}+f_{\eta^{\prime}}^{0} A_{\eta^{\prime}}=\frac{1}{2 \pi^{2}} N_{c} C_{0}+B_{0}+B_{1},
$$

where, for the sake of brevity, we defined different contributions to the ASR as follows,

$B_{0} \equiv N(0,0,0), \quad B_{1} \equiv-\frac{1}{\pi} \int_{0}^{\infty} \operatorname{Im} R(s) d s-\frac{1}{\pi} \int_{s_{0}}^{\infty} \operatorname{Im} F_{3} d s$.

The $B_{0}$ term is the subtraction constant in the dispersion representation of gluon anomaly. The $B_{1}$ term consists of two parts: spectral representation of gluon anomaly and the integral covering higher resonances. The latter is proportional to $\alpha_{s}^{2}$. Indeed, the form factor $F_{3}$ is described by a triangle graph (no $\alpha_{s}$ corrections) plus diagrams with additional boxes ( $\propto \alpha_{s}^{2}$ for the first box term). In the case of both real photons in the chiral limit the triangle amplitude is zero $\left(\propto q^{2}\right)$. So, one can expect $\alpha_{s}^{2}$ suppression of the higher resonances contributions term due to the sufficiently high lower limit of the integral, $s>s_{0} \gtrsim 1 \mathrm{GeV}^{2}$.

Note, that at $s<s_{0}$ there is a NP QCD contribution following from (25): $\langle 0|G \tilde{G}| \gamma \gamma\rangle \propto \frac{\alpha_{e}}{\alpha_{s}}$. So, unlike the second 
term of $B_{1}$ (higher resonances contributions), the first term of $B_{1}$ (spectral part of the gluon anomaly) lies in the essentially nonperturbative region.

Combining the ASRs for the octet (28) and singlet (29) channels of axial current, one gets

$A_{\eta}=\frac{1}{\Delta}\left(\frac{N_{c}}{2 \pi^{2}}\left(C^{(8)} f_{\eta^{\prime}}^{0}-C^{(0)} f_{\eta^{\prime}}^{8}\right)-\left(B_{0}+B_{1}\right) f_{\eta^{\prime}}^{8}\right)$,

$A_{\eta^{\prime}}=\frac{1}{\Delta}\left(\frac{N_{c}}{2 \pi^{2}}\left(C^{(0)} f_{\eta}^{8}-C^{(8)} f_{\eta}^{0}\right)+\left(B_{0}+B_{1}\right) f_{\eta}^{8}\right)$,

where $\Delta=f_{\eta}^{8} f_{\eta^{\prime}}^{0}-f_{\eta^{\prime}}^{8} f_{\eta}^{0}$. Also, making use of the result of the low-energy theorem (26) for $B_{0}$, we can express the two-photon decay amplitudes as follows,

$$
\begin{aligned}
A_{\eta} & =\frac{N_{c} C^{(8)}}{2 \pi^{2} f_{\eta}^{8}}-\frac{B_{1} f_{\eta^{\prime}}^{8}}{\Delta}, \\
A_{\eta^{\prime}} & =\frac{B_{1} f_{\eta}^{8}}{\Delta}
\end{aligned}
$$

Note that the low-energy theorem leads to the cancellation of the photon anomaly term with subtraction part of gluon anomaly $B_{0}$ in (32), so the amplitude $\eta^{\prime} \rightarrow \gamma \gamma$ (in the chiral limit) is entirely determined by $B_{1}$, which is (predominantly) the spectral part of the gluon anomaly.

Let up pass to the numerical analysis. The $B_{0}+B_{1}$ term can be evaluated directly from the Eq. (29) if we use the values of the two-photon decay widths and decay constants of the mesons. The low-energy theorem additionally gives estimation for $B_{0}$, so combining (28), (29), we can separately evaluate $B_{0}$ and $B_{1}$.

For the decay constants $f_{M}^{i}$ we employ the sets of decay constants obtained in different analyses based on the octetsinglet (OS) mixing scheme [14], quark-flavor mixing scheme $[14,38]$ and scheme-free approach $[14,39]$. The results are shown in the Table I.

These results demonstrate, that the contribution of the gluon anomaly and the higher order resonances (expressed by $B_{0}+B_{1}$ term) to the 2-photon decay amplitudes appears to be rather small numerically in comparison with the contribution of electromagnetic anomaly $\left(1 / 2 \pi^{2}\right) N_{c} C^{(0)} \simeq$ 0.058. In fact, these processes are dominated by the electromagnetic anomaly: the electromagnetic part (the first two terms in (31), (32)) makes $95 \%$ and $90 \%$ for $\eta$ and $\eta^{\prime}$ meson decay amplitudes, respectively, while the gluon anomaly originated part (the last two terms $\propto\left(B_{0}+B_{1}\right)$ ) makes only $5 \%$ and $10 \%$ (for the decay constants scheme-free analysis from [14]). Let us note, that this conclusion is valid for the processes with real photons: for the processes involving virtual photons (photon-meson transitions) it may not be true.

Using the low-energy theorem gives the values of $B_{0}$ (subtraction constant) and, in combination with the results
TABLE I. Gluon anomaly term contributions for different sets

\begin{tabular}{|c|c|c|c|c|}
\hline & $\left(\begin{array}{ll}f_{\eta}^{8} & f_{\eta^{\prime}}^{\circ} \\
f_{\eta}^{0} & f_{\eta^{\prime}}^{0}\end{array}\right) \frac{1}{f_{\pi}}$ & $B_{0} \times 10^{2}$ & $B_{1} \times 10$ & $\left(B_{0}+B_{1}\right) \times 10^{2}$ \\
\hline [14], scheme-free & $\left(\begin{array}{cc}1.11 & -0.42 \\
0.16 & 1.04\end{array}\right)$ & -5.55 & 4.91 & -0.64 \\
\hline $\begin{array}{l}\text { [14], OS mix. } \\
\text { scheme. }\end{array}$ & $\left(\begin{array}{cc}0.85 & -0.22 \\
0.20 & 0.81\end{array}\right)$ & -5.36 & 3.84 & -1.53 \\
\hline $\begin{array}{l}\text { [14], QF mix. } \\
\text { scheme }\end{array}$ & $\left(\begin{array}{cc}1.38 & -0.63 \\
0.18 & 1.35\end{array}\right)$ & -5.58 & 6.39 & 0.81 \\
\hline [39], scheme-free & $\left(\begin{array}{cc}1.39 & -0.59 \\
0.054 & 1.29\end{array}\right)$ & -5.77 & 5.86 & 0.095 \\
\hline $\begin{array}{l}\text { [38], QF mix. } \\
\text { scheme }\end{array}$ & $\left(\begin{array}{cc}1.17 & -0.46 \\
0.19 & 1.15\end{array}\right)$ & -5.51 & 5.47 & -0.047 \\
\hline
\end{tabular}
of meson decay constants.

of the ASR (29), $B_{1}$ (dominated by the term $\int_{0}^{\infty} \operatorname{Im} R d s$, the higher resonances term is suppressed as $\propto \alpha_{s}^{2}$, as we noted before). Numerically, $B_{0}$ and $B_{1}$ appear to be rather large: they are of order of the electromagnetic anomaly term. At the same time, $B_{0}$ and $B_{1}$ enter the ASR with different signs and almost cancel each other, giving only a small total contribution to the two-photon decay widths of the $\eta$ and $\eta^{\prime}$. Our conclusions hold for different sets of decay constants which were obtained in independent analyses. ${ }^{2}$

\section{CONCLUSIONS AND OUTLOOK}

Employing the dispersive approach to axial anomaly in the singlet current, we have obtained the sum rule with electromagnetic and gluon anomaly contributions. The gluon contribution consists of a spectral part (originated from $p^{2}$-dependent term) and a subtraction constant (independendent of $p^{2}$ ).

The low-energy theorem was generalized for the case of mixed $\eta-\eta^{\prime}$ states and applied to evaluate the matrix element $\langle 0|G \tilde{G}| \gamma \gamma\rangle$ in the limit $p^{\mu}=0$. It gave an estimation for the subtraction constant of the gluon anomaly contribution in the dispersive form of axial anomaly.

The spectral part of the gluon anomaly was estimated using the ASR in the singlet current and low-energy theorem result for the subtraction part. Numerically, it is found to be significant — of the order of the electromagnetic anomaly contribution. However, it is almost canceled out by the subtraction term of gluon anomaly, resulting in the overall small contribution of the gluon anomaly to the $\eta\left(\eta^{\prime}\right) \rightarrow \gamma \gamma$ decays.

Also, application of the low-energy theorem showed that the two-photon decay of $\eta^{\prime}$ meson (in the chiral limit) is mainly determined by the spectral part of gluon anomaly.

\footnotetext{
${ }^{2}$ Somewhat different results for the constants of octet-singlet mixing scheme [14] can be attributed to rather restricted properties of this scheme. Historically, it was the first one used for the $\eta-\eta^{\prime}$ mixing description, but now it is rarely applied where precise analysis of the processes with $\eta-\eta^{\prime}$ mixing is required.
} 
The smallness of the gluon contribution to radiative decays of pseudoscalar mesons may result in a relative suppression of the $\eta$ and $\eta^{\prime}$ production from the color glass condensate in heavy ion collisions in favor of heavy glueballs. The properties of such glueballs may be deduced in a further analysis of the ASR (20).

\section{ACKNOWLEDGMENTS}

We are thankful to A. Kataev, M. Polyakov, and N. Stefanis for useful discussions and illuminating comments. This work is supported in part by Heisenberg-Landau Program HL-2018 and by RFBR Grant No. 17-02-01108.
[1] S. L. Adler, Axial vector vertex in spinor electrodynamics, Phys. Rev. 177, 2426 (1969).

[2] J. S. Bell and R. Jackiw, A PCAC puzzle: $\pi^{0} \rightarrow \gamma \gamma$ in the sigma model, Nuovo Cimento A 60, 47 (1969).

[3] A. M. Bernstein and B. R. Holstein, Neutral pion lifetime measurements and the QCD chiral anomaly, Rev. Mod. Phys. 85, 49 (2013).

[4] B. L. Ioffe and A. G. Oganesian, Axial anomaly and the precise value of the $\pi^{0} \rightarrow 2 \gamma$ decay width, Phys. Lett. B 647, 389 (2007).

[5] A. D. Dolgov and V. I. Zakharov, On conservation of the axial current in massless electrodynamics, Nucl. Phys. B27, 525 (1971).

[6] B. L. Ioffe, Axial anomaly: The modern status, Int. J. Mod. Phys. A 21, 6249 (2006).

[7] J. Horejsi, On dispersive derivation of triangle anomaly, Phys. Rev. D 32, 1029 (1985).

[8] J. Horejsi and O. Teryaev, Dispersive approach to the axial anomaly, the t'Hooft's principle and QCD sum rules, Z. Phys. C 65, 691 (1995).

[9] O.L. Veretin and O. V. Teryaev, Axial anomaly at the arbitrary external momenta, Yad. Fiz. 58, 2266 (1995) [Phys. At. Nucl. 58, 2150 (1995)].

[10] Y. N. Klopot, A. G. Oganesian, and O. V. Teryaev, Axial anomaly as a collective effect of meson spectrum, Phys. Lett. B 695, 130 (2011).

[11] Y. N. Klopot, A. G. Oganesian, and O. V. Teryaev, Axial anomaly and mixing: From real to highly virtual photons, Phys. Rev. D 84, 051901 (2011).

[12] Y. Klopot, A. Oganesian, and O. Teryaev, Quark-hadron duality, axial anomaly and mixing, JETP Lett. 94, 729 (2012).

[13] D. Melikhov and B. Stech, Universal behaviour of the $\gamma^{*} \gamma \rightarrow\left(\pi^{0}, \eta, \eta^{\prime}\right)$ transition form factors, Phys. Lett. B 718, 488 (2012).

[14] Y. Klopot, A. Oganesian, and O. Teryaev, Transition form factors and mixing of pseudoscalar mesons from anomaly sum rule, Phys. Rev. D 87, 036013 (2013); Erratum, Phys. Rev. D 88, 059902(E) (2013).

[15] A. G. Oganesian, A. V. Pimikov, N. G. Stefanis, and O. V. Teryaev, Matching lightcone- and anomaly-sum-rule predictions for the pion-photon transition form factor, Phys. Rev. D 93, 054040 (2016).

[16] Y. Klopot, A. Oganesian, and O. Teryaev, Axial anomaly and vector meson dominance model, JETP Lett. 99, 679 (2014).
[17] S. S. Agaev, V. M. Braun, N. Offen, F. A. Porkert, and A. Schäfer, Transition form factors $\gamma^{*} \gamma \rightarrow \eta$ and $\gamma^{*} \gamma \rightarrow \eta^{\prime}$ in QCD, Phys. Rev. D 90, 074019 (2014).

[18] N. G. Stefanis, A. P. Bakulev, S. V. Mikhailov, and A. V. Pimikov, Can we understand an auxetic pion-photon transition form factor within QCD?, Phys. Rev. D 87, 094025 (2013).

[19] S. V. Mikhailov, A. V. Pimikov, and N. G. Stefanis, Systematic estimation of theoretical uncertainties in the calculation of the pion-photon transition form factor using light-cone sum rules, Phys. Rev. D 93, 114018 (2016).

[20] A.E. Dorokhov and E. A. Kuraev, Pion transition form factor in the constituent quark model, Phys. Rev. D 88, 014038 (2013).

[21] H. M. Choi, H. Y. Ryu, and C. R. Ji, Spacelike and timelike form factors for the $\left(\pi^{0}, \eta, \eta^{\prime}\right) \rightarrow \gamma^{*} \gamma$ transitions in the lightfront quark model, Phys. Rev. D 96, 056008 (2017).

[22] D. Gomez Dumm, S. Noguera, and N. N. Scoccola, $\eta-\gamma$ and $\eta^{\prime}-\gamma$ transition form factors in a nonlocal NJL model, Phys. Rev. D 95, 054006 (2017).

[23] S. J. Brodsky, F. G. Cao, and G. F. de Teramond, Meson transition form factors in light-front holographic QCD, Phys. Rev. D 84, 075012 (2011).

[24] P. Roig, A. Guevara, and G. López Castro, $V V^{\prime} P$ form factors in resonance chiral theory and the $\pi-\eta-\eta^{\prime}$ lightby-light contribution to the muon $g-2$, Phys. Rev. D 89, 073016 (2014).

[25] S. N. Nedelko and V.E. Voronin, Influence of confining gluon configurations on the $P \rightarrow \gamma^{*} \gamma$ transition form factors, Phys. Rev. D 95, 074038 (2017).

[26] H. Czyż, P. Kisza, and S. Tracz, Modeling interactions of photons with pseudoscalar and vector mesons, Phys. Rev. D 97, 016006 (2018).

[27] R. Escribano, P. Masjuan, and P. Sanchez-Puertas, $\eta$ and $\eta^{\prime}$ transition form factors from rational approximants, Phys. Rev. D 89, 034014 (2014).

[28] R. Escribano, S. Gonzàlez-Solís, P. Masjuan, and P. Sanchez-Puertas, $\eta^{\prime}$ transition form factor from space- and timelike experimental data, Phys. Rev. D 94, 054033 (2016).

[29] C. Hanhart, A. Kupśc, U.-G. Meißner, F. Stollenwerk, and A. Wirzba, Dispersive analysis for $\eta \rightarrow \gamma \gamma^{*}$, Eur. Phys. J. C 73, 2668 (2013); Erratum, Eur. Phys. J. C 75, 242(E) (2015).

[30] L. Rosenberg, Electromagnetic interactions of neutrinos, Phys. Rev. 129, 2786 (1963). 
[31] V. L. Eletsky, B. L. Ioffe, and Y. I. Kogan, The $g_{\omega \rho_{\pi}}$ coupling constant from Qcd sum rules, Phys. Lett. 122B, 423 (1983).

[32] A. V. Radyushkin and R. T. Ruskov, Transition form-factor $\gamma \gamma^{*} \rightarrow \pi^{0}$ and QCD sum rules, Nucl. Phys. B481, 625 (1996).

[33] A. S. Gorsky, B. L. Ioffe, and A. Y. Khodjamirian, New anomaly: Nonvanishing emission and scattering of longitudinal photons in massless quantum electrodynamics, Phys. Lett. B 227, 474 (1989).

[34] A. V. Efremov and O. V. Teryaev, On renormalization of axial anomaly, Yad. Fiz. 51, 1492 (1990) [Sov. J. Nucl. Phys. 51, 943 (1990)].
[35] M. A. Shifman, Anomalies in gauge theories, Phys. Rep. 209, 341 (1991).

[36] D. J. Gross, S. B. Treiman, and F. Wilczek, Light quark masses and isospin violation, Phys. Rev. D 19, 2188 (1979).

[37] B. L. Ioffe, Masses of light quarks and interaction of lowenergy eta mesons, Yad. Fiz. 29, 1611 (1979) [Sov. J. Nucl. Phys. 19, 827 (1979)].

[38] T. Feldmann, P. Kroll, and B. Stech, Mixing and decay constants of pseudoscalar mesons, Phys. Rev. D 58, 114006 (1998).

[39] R. Escribano and J. M. Frere, Study of the eta-eta-prime system in the two mixing angle scheme, J. High Energy Phys. 06 (2005) 029. 